\title{
ASTROPHYSICS
}

DOI: http://dx.doi.org/10.18524/1810-4215.2018.31.144437

\section{POSITRON ANNIHILATION SPECTROSCOPY OF YOUNG SUPERNOVA REMNANTS}

\author{
D.N. Doikov \\ Odessa National Maritime University, Dep. of Mathematics, Physics and Astronomy \\ doikov@mail.bg
}

\begin{abstract}
This paper discusses radiative and thermodynamic properties of cold rarefied aggregates of nonuniformly distributed gas and dust through which high fluxes of positrons with energies of 0.011-3.6 MeV pass. The investigated gas is in the form of a nebula with densities of $1-10^{8} \mathrm{~cm}^{-3}$ and temperatures ranging from $30-100$ $\mathrm{K}$. We estimated the energy input into thermodynamic temperatures of the ejecta components. Additional heating and $\gamma$-ray luminosity of all the components of the examined ejecta were factored in. The structure of the radiation field $\mathrm{S}(\mathrm{E}, \mathrm{r})$ and electron velocity distribution function $\mathrm{F}(\mathrm{E}, \mathrm{r})$ were determined depending on the energies of quanta and electrons, respectively. The ejecta of SN 1987A was considered as an ideal object to investigate the positron impact on the nebula. The interaction of positrons with solids, atoms and molecules was examined separately. Traveling of positrons in solids typically results in their enhanced amorphous state, heating and annihilation with free electrons of solid-state grains with emission of two photons each of the energy $\mathrm{E}_{\gamma}=0.511 \mathrm{MeV}$. Consequent-
\end{abstract} ly, a characteristic excessive luminescence of dust particles, namely astronomical silicates and graphite, occurs. In fact, the energy loss of fast positrons due to ionisation leads to consecutive creation of K-L-M vacancies, which is followed by cascade transitions with the transfer of the remaining energy to the Auger electrons rather than to surrounding atoms which make up a solid. In this case, in a solid particle, the energy released in cascade transitions is either used to change the lattice structure or converted into $\gamma$-quanta emission upon the annihilation of positrons and K-L electrons. The estimated energy used to heat a solid particle itself makes up a half of the energy released in the downward M-L-K cascades, which is indicative of a significant contribution to the energy balance of the ejecta dust. This contribution exceeds the energy estimates from the net radiation loss of particles in the matter. In the nebular atomic-molecular plasma of the supernova ejecta, the conservation of energy dictates that the energy lost to create a K-vacancy is allocated to the subsequent cascade transitions and the Auger electrons while the one-photon annihilation of positrons and K-electrons of atoms and molecules yields characteristic $\gamma$-quanta with energies close to their own values $-\mathrm{E}_{\gamma} \approx 1.022 \mathrm{MeV}$. The study of annihilation of positrons and K-electrons with emission of a single photon revealed a relationship between the energy of the emitted $\gamma$-quanta with the recoil energies of nuclei $E_{n}$, binding energies of K-electrons of different atoms $-E_{b}$ and energies of incident positrons $E_{p}$. The cross-sections for interactions of positrons and atoms in the investigated young supernova remnants were calculated and scaling of electron arrangements of the atoms involved in those interactions was performed. We pointed out that for the iron-peak atoms the cross-section for the positron-atom interaction increases by four orders of magnitude, thus making the interaction between positrons and electrons in the K-L shells the most probable. It is shown that in astrophysics the positron annihilation spectroscopy of matter yielding a characteristic radiative response has opened up new opportunities for studying young supernova remnants and active galactic nuclei. In particular, it is now possible to independently determine the mass ratio of dust and gasmolecular components by the strength ratio of annihilation $\gamma$-lines I (0.511)/I (1.022). We report the estimated contribution of various elements to the profiles of the indicated $\gamma$-lines and conclude that it is not related to the proper dynamic motions of the supernova remnants. This is a crucial factor in the study of bulges in active galactic nuclei wherein the physical conditions are assumed to be steady-state in relatively large scales.

Keywords: II type SN remnants, radioactive elements, positron spectroscopy of remnants dust and gas.

АБСТРАКТ. В даній роботі розглянуто радіаційні і термодинамічні властивості холодних розріджених агрегатів, які містять неоднорідно розподілені газ і пил, що зазнають інтенсивного проходження потоків позитронів з енергіями 0.011-3.6 МеВ. Розглянутий газ перебуває в небулярному стані зі щільністю $1-10^{8} \mathrm{~cm}^{-3}$ i температурою 30-100К. Отримано енергетичний внесок у термодинамічну температуру компонентів оболонок. Враховано енергетичний внесок у додаткове нагрівання і світіння в $\gamma$-області спектру усіх компонентів випромінювання оболонок. Визначено структуру поля випромінювання S(E,r) i функцію розподілу електронів за швидкостями $\mathrm{F}(\mathrm{E}, \mathrm{r})$ в залежності від енергій квантів або електронів. Оболонку SN 1987A розглянуто як ідеальний об'єкт впливу позитронів на небулярне середовище. Окремо розглянуто взаємодії позитронів з пилом, атомами і молекулами. Характерними наслідками руху позитронів у твердих частках є збільшення їх аморфності, нагрів i двухфотонна анігіляція 3 вільними електронами твердотільних утворень енергією квантів $E_{\gamma}=0,511 \mathrm{MeV}$, внаслідок чого виникає характерне надлишкове світіння пилу - астрономічних силікатів і графіту. Іонізаційні втрати енергії швидких позитронів по суті не 
призводять до послідовних процесів утворення K-L-M вакансій, каскадних переходів і передачі залишкової енергії Оже-електронам, а викликають нагрів пилу. У цьому випадку енергія від каскадних переходів у твердій частці більшою мірою пов'язана з витратами на зміну кришталевої структури і будують $\gamma$-кванти від анігіляції позитрона 3 K-L-електронами. Енергію, витрачену саме на нагрів пилу, оцінено як половина енергії N-M-K каскадів. У цьому випадку знайдена суттєва складова у енергобаланс пилових часток оболонок, яка перевищує оцінки по чистим радіаційним втратам часток у речовині. В небулярній атомномолекулярній плазмі оболонки наднової енергія, яка утворена позитроном К-вакансії перерозподіляється між каскадними переходами і Оже-електронами, а однофотонна анігіляція позитронів 3 К-електронами атомів і молекул продукує характерні $\gamma$-кванти з енергіями значень близько $E_{\gamma} \approx 1,022 \mathrm{MeB}$. В роботі, під час дослідження однофотонної анігіляції позитронів 3 К-електронами виявлено зв'язок енергій утворених $\gamma$ квантів 3 енергіями віддачі ядер $E_{n}$, енергіями зв'язка електронів з К-оболонок $-E_{b}$ різних атомів і енергіями позитронів $-E_{p}$. Обчислені перетини взаємодій позитронів з атомами розглянутих молодих оболонок наднових і отримані масштаби атомних електронних структур, що залучено у цю взаємодію. Визначено, що для атомів залізного піку перетини взаємодій позитронів 3 атомами зростає на 4 порядки і утворює взаємодії позитронів 3 оболонок K-L-електронів найбільш вірогідними. Доведено, що позитронна спектроскопія речовини у вигляді його характерного радіаційного відгуку розкриває для астрофізики нові можливості у дослідженні молодих залишків наднових та активних ядер галактик. Зокрема, у відношенні інтенсивності анігіляційних $\gamma$-ліній $\mathrm{I}(0,511) / \mathrm{I}(1,022)$ виявляється можливим незалежне визначення відношення пилової маси до маси газово-молекулярної складової. Зроблено висновки 3 приводу вкладу у профілі зазначених $\gamma$ ліній, які викликано різними елементами. Показано, що цей внесок не пов'язаний $з$ власними динамічними рухами оболонок наднових. Вищезазначена обставина $€$ особливо важливою при дослідженні Балджа активних ядер галактик, де фізичні умови вважаються стаціонарними на відносно великих масштабах

Ключові слова: Наднові II типу, радіоактивні елементи, позитрона спектроскопія газу та пилу залишків наднових.

\section{Introduction}

The motion of fast particle streams through matter has always been used for studying of media. In the age of the first experiments of Ernest Rutherford and his apprentices, it had been already evident that the quantum structure of matter reacted differently to such particles. In those experiments, the motion of fragments of radioactive nuclei after their decay could trigger different energy responses for one and the same matter, depending on its phase state. For solids and even for liquids, the loss of energy results in the least radiative response. After a fragment resulted from the decay creates a vacant K-L-M energy level in an atom in a solid phase, the energy conserved in this transition to the excited state is re-allocated between consecutive cascades and change of the lattice structure of the solid. In addition, the stronger the interatomic bond in a solid is, the more efficient the latter route is. For a perfect diamond-lattice the radioactive emission results in the change of its spectral properties (i.e. colour, absorption coefficient, conductivity, Young's modulus, etc.). This is why dust particles in young supernova remnants change their optical and structural properties even during relatively short observations. A decrease in Young's modulus is indicative of their amorphisation, as well as accelerated disintegration and sputtering of these particles in the interstellar medium. Liquids behave similarly to solids, with the only exception being that the released energy is converted predominantly into emission and dissociation of molecules which leads to an effective increase in the temperature of the liquid. In astrophysics, the nebulae have never been observed as liquid gas-and-dust aggregates. Therefore, it is more reasonable to focus on studying the radiative properties of gas and dust being traversed by positrons. The presence of strong fluxes of hard radiation in the interstellar gas, supernova remnants and upper atmosphere of some stars results in qualitatively new effects and appearance of spectra of these media. In such media, as a result of vacant $\mathrm{K}$ energy levels created due to positron traveling, atoms undergo consecutive cascade transitions with the transfer of the remaining energy to the Auger electrons. In some cases, hard radiation produced in supernova remnants passes through very cold $(30-80 \mathrm{~K})$ gas and dust of the nebula. In these circumstances, we have got dual-mode source functions $S(E, r)$ and electron velocity distribution functions $F(E, r)$ depending on the energies $\mathrm{E}$ of photons and electrons, respectively, as well as on the current position $\mathrm{r}$ in the Type II SN 1987A remnant (Jerkstrand et al., 2001; Doikov \& Andrievskii, 2017; Doikov et al., 2018). The low probability of recombination of the excited atoms and molecules, along with the given specific values $S(E, r)$ and $f(E, r)$, indicates that the excited state of atoms of the supernova ejecta due to their ionisation is being maintained. As a consequence, the lifespans of a certain portion of atomic ions are long and comparable with the typical half-lives of radioactive elements injecting respective fast particles. A new method of distinguishing between isotopes using the ratio of integrated strengths of their lines $\mathrm{I}\left({ }_{20}^{44} \mathrm{CaI}\right) / \mathrm{I}\left({ }_{20}^{40} \mathrm{CaI}\right)$ was reported in the afore-mentioned papers. ${ }_{20}^{44} \mathrm{CaII}$ is a final product of radioactive decay chain ${ }_{22}^{44} \mathrm{Ti} \stackrel{85 \mathrm{y}}{\longrightarrow}{ }_{21}^{44} \mathrm{Sc} \rightarrow{ }_{20}^{6 \mathrm{~h}} \mathrm{Ca}$ while ${ }_{20}^{40} \mathrm{CaI}$ is the end-product of synthesis in the progenitor star. Line profiles of neutral and ionised atoms are well studied for both isotopes. Spectroscopic response to the positron fluxes in the ejecta of SN 1987A was observed with the Hubble Space Telescope on $07^{\text {th }}$ of January, 1995, eight years after the supernova explosion. Optical transitions in the neutral and ionised calcium atoms were observed among others (Jerkstrand et al., 2011). Over several years of the infrared studies of this supernova, it has become evident that its ejecta consist of fragments (clumps) in which heavy elements that comprised the progenitor core before the explosion are concentrated 
(Doikov et al., 2018). These clumps contain dust particles which produce mid- and far-IR emission spectra typical for astronomical silicates. Graphite and other carboncontaining particles are less abundant and may have formed in the clumps originated from the $\mathrm{C} / \mathrm{O}$ region of the progenitor star (Woosley et al., 1991).

The mere fact of the optical emission spectra from the ejecta clumps is indicative of the presence of astronomical silicates therein. The inter-clump medium, as well as the frontal regions of the remnant, is rich in hydrogen and helium which together comprise $70 \%$ of the supernova ejecta matter. The density of hydrogen and helium in the clumps in the inner part of the ejecta is $10^{4} \mathrm{~cm}^{-3}$ while in the inter-clump medium it is close to the interstellar value of $1 \mathrm{~cm}^{-3}$. The presence of clumps in the interior of the ejecta is associated with their inertness. Major portion of hydrogen and helium ( $90 \%$ of their total abundance) is concentrated in front of these clumps, i.e. in the outer part of the ejecta. According to Varosi \& Dwek (1999), the minimum number of clumps in the ejecta with the elemental distribution as specified above may be set to 100 . Such distribution of the ejecta fragments, i.e. clumps, was suggested on the basis of high-precision measurements of the IR emission from the dust concentrated in those clumps. Thus, there is a problem set out to solve, which is to semi-empirically determine the spectroscopic response within the clumps containing the radioactive isotope of titanium $-{ }_{22}^{44} \mathrm{Ti}$. The total amount of this isotope derived from the measurements of the radiation component of the products of a series of radioactive transformations ${ }_{22}^{44} \mathrm{Ti} \stackrel{85 y}{\longrightarrow}{ }_{21}^{44} \mathrm{Sc} \stackrel{6 h}{\rightarrow}{ }_{20}^{44} \mathrm{Ca}$ is $10^{27} \mathrm{~kg}$ (Grebenev et al., 2012). With the half-life of 85 years, the standardised number of positrons within the bulk of the ejecta is $10^{51}$. Prior to their annihilation, positrons in the clumps undergo $10^{3}$ collisions on average. In the $\mathrm{H} / \mathrm{He}$ of the outer part of the ejecta, this number averages $10^{4}-10^{5}$ collisions. It means that over last 28 years after the hydrogen recombination the radioactive decay of ${ }_{22}^{44} \mathrm{Ti}$ has been a significant source of excitation of atoms and molecules. This paper focuses on the study of $\gamma$-emission spectra resulted from the positron annihilation in the gas and dust components of young supernova remnants. There are qualitative physical differences in the positron interaction with solid dust fraction and individual atoms and molecules. When a positron moving through matter is slowing down and losing energy by ionisation, it will decelerate until its energy is down to the thermal energy of free or atomic loosely-bound electrons in the solid. As a result, the positron and an electron form a pair which further annihilate with the emission of two $\gamma$-quanta each with the energy $E_{\gamma}=0,511 \mathrm{MeV}$. Another mode of electromagnetic interaction between positrons and bound K-electrons results in the annihilation with emission of a single photon. This process will be discussed below in section 2 which proves its relevance to the study of young supernova remnants after the hydrogen recombination. The dependence of the cross-section for the one-photon annihilation on the energies of incident positrons and main characteristics of atoms and their nuclei will be considered separately in the same section. In this case, given the preliminary determined De Broglie wavelengths for positrons, it is possible to identify the atomic energy levels in which positrons enter into annihilation interactions with electrons. Average thermodynamic quantities of supernova remnants, unlike those of the active galactic nuclei, change with time as described in sub-section 1.2. Time-varying chemical composition due to radioactive decay is another specific feature of such physical systems. Positron annihilation spectroscopy, combined with the findings reported by Doikov et al. (2018), allows obtaining information about the chemical evolution of supernova remnants using $\gamma$-lines produced by different elements. The kinetic energy of recoiling nuclei released upon the investigated single-photon annihilation will be derived in the same section. We suggest considering the relationship between the calculated kinetic energy and characteristics of the recoiling nuclei spectral lines, including their Doppler broadening. These aspects have been discussed in more detail in Doikov et al. (2018). The $\gamma$-line profiles in astrophysical systems of a given type have been understudied; however, the improved accuracy of $\gamma$-detectors along with the results obtained in this research make it possible to adopt them for the quantitative study of diffuse nebulae which include supernova remnants.

\section{Positron annihilation spectroscopy of young su- pernova remnants. Energy loss of positrons due to ionisation without annihilation in the nebular gas.}

The main reason for the luminescence of cold gas and dust in young supernova remnants is the ionisation by positrons moving in the matter. Positrons diffuse the ejecta and trigger the luminescence emission throughout its entire volume which can be observed in different spectral regions at the current epoch. Let us consider such supernova remnant as a closed system with an attached moving reference frame. The law of total charge conservation for the ejecta should imply the equal number of positrons produced and annihilated per unit time. Otherwise, the condition of zero net charge of all ions, electrons and positrons is not met. In the afore-mentioned radioactive decay series, one $1 \mathrm{~s}$ electron of the ${ }_{22}^{44} \mathrm{Ti}$ isotope atom is captured while another one is shed as an Auger electron following the positron emission of the unstable isotope ${ }_{21}^{44} S c$. As noted in Doikov et al. (2017), in the supernova remnants positrons are more likely to annihilate with atomic K-electrons rather than with free thermal electrons. This statement has been corroborated by the International Gamma-Ray Astrophysics Laboratory (INTEGRAL) observations (Grebenev et al., 2012). The energy loss of positrons due to ionisation adds new cascade transitions to the detailed static balance equation; and the Auger effect is factored in in the Saha ionization equation factors. As a result, the supernova remnant is supplemented with the radiation field of hard photons and high-energy Auger electrons. These emergent quanta of hard radiation and especially the ejected Auger electrons proceed to excite the surrounding atoms of metals and give rise to copious number of transitions thereby filling the supernova ejecta with the diffusive radiation. The respective transitions triggered in such a manner were observed in optical and IR regions with the Hubble Space Telescope in January, 1995. At the current epoch, this physical system is at the 
nebular phase. It means that quanta and electrons have energies which are sufficiently high to excite atoms and molecules within the entire volume of the ejecta. Since radioactive decay serves as an extra source of energy, we consider certain elementary steps of the ${ }_{22}^{44} \mathrm{Ti}$ radioactive transformations which are valuable for further calculations of estimates in compliance with the radioactive decay law.

$$
N=N_{0} e^{-\lambda t}=N_{0} e^{-\frac{0.698}{T_{1 / 2}} t}
$$

where $T_{1 / 2}$ is the half-life of ${ }_{22}^{44} T i ; N_{0}$ is the initial number of atoms of this radioactive isotope. It is more efficient to use the rate of decay, i.e. the activity of the radioactive isotope, expressed as A, which is the number of decays per second and equal to the time derivative of $\mathrm{N}$. In other words,

$$
A=\frac{d N}{d t}=-N_{0} \frac{0.698}{T_{1 / 2}} e^{-\frac{0.698}{T_{1 / 2}} t} .
$$

Taking into account the total amount of the titanium isotope ${ }_{22}^{44} \mathrm{Ti}$, which has been estimated from the observations by Grebenev et al. (2012) and is $10^{27} \mathrm{~kg}$, it may be deduced that at the current epoch on average one to three decays fall on each $1 \mathrm{~cm}^{3}$. It means that every second one to three positrons is ejected. The density of matter in the regions where the clumps are located is of the order of $10^{4}$ particles per $1 \mathrm{~cm}^{3}$ or $10^{4} \mathrm{~cm}^{-3}$. In the outer H/He envelope in front of the clumps, the density reaches $10^{8} \mathrm{~cm}^{-3}$. When an ejected positron just starts traveling, its maximum energy is of the order of $3.6 \mathrm{MeV}$; then, it loses $0.1 \%$ of its energy in each collision with the ejecta atoms. The kinetic energy loss of such a positron by ionisation is due to its annihilation with predominantly K-electrons, resulting in the creation of the respective core hole and transition of the atom to a highly excited state.

Prior to annihilation with a bound atomic electron, a positron undergoes at least $10^{3}$ collisions with heavy atoms and up to $10^{4}$ collisions with light atoms. In other words, in the SN 1987A remnant at the current nebular phase, positrons are promoting transitions to the excited states throughout the entire ejecta, rather than just within clumps wherefrom they have been ejected (Doikov et al., 2018). Let us calculate the specific energy of such collisional excitation by positrons. To this end, the chemical composition of the supernova ejecta should be specified. Having defined the elemental distribution of the matter in the ejecta clumps, we can calculate the initial rates of the elementary processes required to comply with the basic laws of conservation. First of all, we should take into account the Kirchhoff's law which states that in thermodynamic equilibrium the emissivity of matter is equal to its absorptivity. The thermal radiation coefficient $\eta(v)$ should be correlated with the coefficient of thermal energy (heat) absorption $k(v)$ and intensity of thermal radiation $I(n, v)$ :

$$
\eta(v)=k(v) I(n, v) .
$$

This formula does not require factoring in the dissipation of energy of incident positrons due to its minor contribution to the kinetic energy dissipation coefficient as compared to the reduction of energy due to positron scat- tering (or deflection). According to the preliminary estimates reported by Kaastra \& Mewe (1993) upon the results of quantum-mechanical computations, for the irongroup elements about a half of the energy conserved upon the creation of a K-L vacancy is spent on triggering a cascade of radiative transitions while the rest is converted into the kinetic energy of the Auger electrons. In this case, equation (3) may be written as follows:

$$
\eta(v)=0,5 k(v) I(n, v)
$$

The energy carried away by the Auger electrons should be then reduced by the total ionisation potential of all principal energy levels which these electrons occupied before the ejection. (Figures 1-3 in Doikov et al. (2018) illustrate the results of precise calculations of kinematic and radiative properties of the ejecta for different regions of the SN 1987A remnant, given its clumpiness as postulated by Woosley et al. (1988). The spectroscopy of nebulae is feasible if there is a central compact source of radiation or if the nebula itself contain any sources of excitation of radiation components (gas, molecules and dust). Among various codes used to simulate conditions in planetary nebulae, the most popular is the Cloudy code developed by Ferland (1998) and its modifications by Melech (2015). Note that the incoming flux of hard radiation from the central source to the planetary nebula does not affect the charge neutrality of the medium. Hard radiation promotes both light elements and metals to multiple excited states. In other words, one hard UV quantum when hitting, for instance, an iron atom, promotes several low-lying excited states. Subsequently, the de-excitation of the atom occurs via numerous allowed transitions through intermediate excited states. The nebula eventually luminesces producing a characteristic emission spectrum. The progress in examining spectra of planetary nebulae made it possible to accurately specify their physical properties and distinguish the CNO (carbon-nitrogenoxygen) ions sensitive to the electron density and temperature, as well as to other important characteristics of the nebula. In particular, the fluxes and spectra produced by the central source can be adequately separated from the secondary, the so-called diffuse components of the spectra of planetary nebulae. When the sources of excitation are present in the ejecta being diffusively distributed therein, it seems feasible to study it using the Osterbrock-Lucy equations (formulae 8-12 here below). Employment of these formulae requires determination of the cross-sections for absorption, dissipation and emission in the nebular gas of the ejecta, as well as densities of atoms of different chemical elements and geometric parameters of the clumpy structure of the ejecta.

Earlier mentioned cascade transitions in atoms throughout the ejecta are constrained on their characteristics by the selection rules. Basically, there is only one allowed electron transition to the $\mathrm{K}$ shell $(2 \mathrm{p}-1 \mathrm{~s})$ in the $\mathrm{CNO}$ elements. In the iron-peak elements after Sc, two electron transitions are allowed $(3 d-2 p$ and $2 p-1 s)$. In other atoms, if the vacancy-filling transition is forbidden, the vacant energy level can only be filled by the recombination through allowed intermediate transitions (e.g. through f-levels) rather than via cascade transitions. At the current epoch, the primary radiation field is formed with- 
out significant conversion of the short-wave quanta emission to optical one, with the only exception being the intrinsic IR-emission of the ejecta dust particles, which are astronomical silicates. According to the findings by Doikov et al. (2018), the presence of the graphite dust in the SN 1987A debris should have resulted in blocking out the optical radiation, though, in fact, it was recorded during the Hubble Space Telescope observations in 2005, in the eighth year after the supernova explosion.

3. Young supernova remnants. Gas dynamics in the supernova ejecta.

Today, the most extensively studied supernova remnant is SNR 1987A. As all supernova remnants, it has already passed the stage of free expansion after the supernova explosion 31 year ago, and is reckoned to be in the form of a nebula. Due to exponential relationship between the key thermodynamic and kinematic parameters of the supernova ejecta, the gas-dynamic structure of intense explosions during the adiabatic phase of its expansion makes it possible to describe the remnant by the similarity and dimensional methods. In so doing, given a sufficient set of observations, the power-law indices in the equations of gas dynamics for the spherically expanding ejecta can be determined semi-empirically and written as the following simple expressions:

$$
\begin{gathered}
\rho\left(M_{r}, t\right)=\rho\left(M_{0}, t_{0}\right)\left(\frac{t}{t_{0}}\right)^{-3} \\
T\left(M_{r}, t\right)=T\left(M_{0}, t_{0}\right)\left(\frac{t}{t_{0}}\right)^{-3(1-\gamma)}
\end{gathered}
$$

where $M_{0}=20 M_{\odot}, \gamma=1.25-1.30$. The temperature $T\left(M_{r} t\right)$ and density $\left(M_{r}, t\right)$ of the ejecta depends on the distance from the explosion site $r$ and timescale of the process $\boldsymbol{t}$. With the knowledge of the law of variations in the mean thermodynamic values it became possible to calculate the amount of dust resulted from the ejecta expansion. According to various estimates, the mass of the dust reaches one solar mass. Assuming the clumpy stricture of the supernova ejecta, it may be deduced that astronomical silicates and graphite dust were formed in different regions of the ejecta at different times (within one to two years after the explosion as per observations). The presence of the dust particles, which radiate via luminescence being exposed to the emission from the shock fronts, made it possible to study the interstellar medium at the front of the forward shock, as well as the supernova ejecta undergoing shock cooling described by formulae (5), over two decades after the explosion. However, reduced X-ray emission from the forward shock, which has been observed since 2015, should have resulted in the disappearance of the intrinsic emission of the dust; though, in fact, the X-ray emission has decreased to a lesser extent than predicted by the calculations of the dust energy balance. The self-emission of gas and dust in the ejecta observed over last 20 years has called for the presence of extra sources of energy, to which, earlier in the introduction, we have attributed the radioactive transformations of the ${ }_{22}^{44} \mathrm{Ti}$ isotope. Thus, for the supernova ejecta we can postulate the presence of a nebula with inner sources of energy. Observations in recent years have also shown that the supernova ejecta are clumpy. Dust and heavy elements, which are the supernova nucleosynthesis products, are concentrated in the ejecta clumps. The number of such clumps is of the order of 100 . The clumps are surrounded by hydrogen and helium; these elements being concentrated mainly in the outer regions of the ejecta make a significant fraction of its total mass.

The mean relative abundances of chemical elements in this study were estimated from the pair of equations (5). The total number of positrons in the ejecta is $10^{51}$, which is equivalent to one positron per $1 \mathrm{~cm}^{3}$. The volume of the ejecta expands with time while the number of positrons per $1 \mathrm{~cm}^{3}$ of the ejecta decreases; in any case, it results in the reduction in the emissivity within the entire bulk of the ejecta. The total luminosity of the ejecta may be taken as a constant value, which is valuable for the observations at short wavelengths, including the $\gamma$-rays. Another important aspect is that the shape of the remnant is not of significance for the integrated observations of sufficiently distant young supernova remnants. In this case, the remnant is considered to be spherically symmetric. It is also important for mapping the supernova ejecta.

\section{Radiative transfer in the ejecta}

A specific feature of the Type II supernova remnants is the distribution of emitters within the entire bulk of the ejecta clumps. In this case, the motion of radiative fluxes is diffusive and isotropic rather than directed. This problem has been first considered for the active galactic nuclei. The excited state of atoms, molecules and dust resulting in their own radiation within large volumes of gas-and-dust aggregates was associated with the ejection of high-energy particles, i.e. cosmic rays, and exposure to hard X-rays and $\gamma$-rays. A solution to the equation of radiative transfer for volumetrically distributed emitters, i.e. dust particles, was proposed in the monograph by Osterbrock (1989). Later, Lucy et al (1991) modified the relations obtained by Osterbrock in order to simultaneously factor in the absorption and dissipation of the volumetrically emitted quanta. The resulting relations, which are important for our study, bear the name the Osterbrock-Lucy equations:

$$
\begin{gathered}
\mathcal{P}_{\text {esc }}^{\text {unif }}(\tau, \omega) \equiv \frac{P_{e}(\tau)}{1-\omega\left[1-P_{e}(\tau)\right]}, \\
P_{e}(\tau)=\frac{3}{4 \pi}\left[1-\frac{1}{2 \tau^{3}}+\left(\frac{1}{\tau}+\frac{1}{2 \tau^{2}}\right) e^{-2 \tau}\right] \\
\text { where } \tau_{\text {ext }}=\tau_{\text {abs }}+\tau_{\text {scat }}, \omega=\tau_{\text {scat }} / \tau_{\text {ext }} .
\end{gathered}
$$

Assuming that $\varepsilon$ is the emission per unit volume of a clump and adopting equations (18) and (19), Varosi \& Dwek (1998) derived formulae of the intensity $I_{\text {out }}(\tau, \theta)$ and total emerging flux $F_{\text {out }}(\tau)$, which will be convenient for further calculations:

$$
\begin{aligned}
& I_{\text {out }}(\tau, \theta)=\frac{\varepsilon}{\rho \kappa}\left(1-e^{-2 \tau \cos (\theta)}\right), \\
& F_{\text {out }}(\tau)=\frac{\pi \varepsilon}{\rho \kappa}\left(1-\frac{1}{2 \tau^{3}}+\left(\frac{1}{\tau}+\frac{1}{2 \tau^{2}}\right) e^{-2 \tau}\right)
\end{aligned}
$$

For the ejecta clumps with different composition the total emerging flux $F_{\text {out }}(\tau)$ depends on the ratio between 
the scattering, absorption and emission coefficients. The structure of the radiation field of clumps containing no dust was described by Doikov et al. (2018). In particular, the source function S(E,r) was defined. The presence of a substantial amount of the graphite dust in the ejecta should have noticeably suppressed the short-wavelength portion of the source function $S(E, r)$ that could have made the production of the remnant atomic spectra, de facto observed in 1995, impossible. The graphite dust contributes to the emission from the shock fronts of SN 1987A whereas the silicate dust emission fits better to explain the spectroscopic features of the ejecta. The particle-size distribution function $\mathrm{n}(\mathrm{a}) \approx a^{-3,5} ; 10^{-9} m<a<0.25$. $10^{-6} \mathrm{~m}$ is typical for the silicate dust. When interpreting the spectroscopic data on the interstellar medium and supernova remnants, we consider the so-called astronomical silicates. Apart from their intrinsic emission, these particles scatter the short-wavelength component of S(E,r) and let the optical and partially IR emission through. When calculating monochromatic optical depth of a clump for a given $\mathrm{S}(\mathrm{E}, \mathrm{r})$, we can add the expression for the emerging flux produced exclusively by astronomical silicates to formula (21) for the total emerging flux $F_{\text {out }}(\tau)$ :

$$
F_{\lambda}^{s i l}(\lambda)=4 \pi m_{d} k(\lambda) B_{\lambda}\left(T_{d}\right)
$$

In this formula, $m_{d}$ is the mass of the silicate dust in an ejecta clump; $k(\lambda)$ is the absorption coefficient for a given type of astronomical silicates; $B_{\lambda}\left(T_{d}\right)$ is the Planck function for particles with the temperature $T_{d}$. Unlike active galactic nuclei wherein particles which promote excitation, as well as quanta, widely range in their energies, the SN 1987A ejecta contain positrons serving as an excitation source with energies constrained by the nuclear transmutation laws which can reach $3.6 \mathrm{MeV}$. As mentioned earlier, the satellite observations provided a rather accurate estimate of the amount of the titanium radioactive isotope in the SN $1987 \mathrm{~A}$ ejecta $-10^{27} \mathrm{~kg}$. It means that the energetic activity of the radioactive titanium is strictly determined at a given instant of time. The spectroscopic response of cold gas and dust in the ejecta, through which the fluxes of positrons pass, is no different from the analogous spectroscopic response to the flux of electrons. The differences appear at low intrinsic energies of positrons, i.e. at the stage of positron annihilation. Since the density of matter of the ejecta at the nebular phase is exceptionally low, it may be postulated that the probability of annihilation of positrons and free electrons within this nebula is low. The electron density is orders of magnitude lower than the atomic one. Hence, the annihilation of a positron with a bound atomic electron would be more probable. Low-energy positrons within the ejecta clumps annihilate mainly with K-L electrons of atoms. As will be shown below, upon such annihilation event, following the laws of conservation of energy and momentum, the atom is allowed to emit a single $\gamma$-quantum with the energy of the order of 1.0-2.79 MeV. Annihilation of positrons with free electrons yields two $\gamma$-quanta each with the energy of $0.511 \mathrm{MeV}$. It is the positron annihilation with bound electrons resulting in the emission of a $1.022 \mathrm{MeV}$ quantum that has been detected by the INTEGRAL observations. It is problematic to suppose that there is a stable production of a certain amount of primary electrons with the given narrow range of energies similar to that one of positrons in the absence of a compact relativistic source in the supernova remnant. Therefore, young supernova remnants may be considered as unique objects with respect of their $\gamma$-ray spectroscopic measurements. The improved resolution of the $\gamma$-spectra of young supernova remnants allows considering the problem of differentiation of these spectra by the chemical composition. The optical thickness of matter factoring in the energy distribution of quanta in the $\gamma$-ray region of the ejecta spectrum will be derived in the next section.

5. Determination of the SN composition by the positron annihilation spectroscopy methods. Annihilation $\gamma$-lines.

The determination of the field of positron velocities (kinetic energies) in the ejecta, as well as the elemental electronic arrangement, enables us to set the problem of their identification. The radioactive transformation of the ${ }_{22}^{44} \mathrm{Ti}$ yields positrons with low Lorenz factor. In this case, the problem on the interaction and further annihilation of a positron with a K-electron of the target atom can be solved in the boundary approximation of low energies (Doikov et al., 2018; Mikhaylov et al., 1998; Anshu et al. 2010). To this end, first, it is necessary to calculate the cross-section for the single-photon annihilation of a positron with an atomic K-electron by the following formula:

$$
\begin{aligned}
& \sigma_{e^{+}}^{+}=\sigma_{0} Z^{5} \alpha^{4} \varphi\left(E_{e^{+}}\right), \\
& \varphi\left(E_{e^{+}}\right)=\frac{4 m_{n}^{3}}{p_{e^{+}}\left(E_{e^{+}}+m_{e}\right)}\left(\frac{E_{e^{+}}^{2}}{m_{e}^{2}}+\frac{2}{3} \frac{E_{e^{+}}}{m_{e}}+\frac{4}{3}-\right. \\
& \left.-\frac{E_{e^{+}+2 m_{e}}}{p_{e^{+}}} \ln \frac{E_{e^{+}+p_{e^{+}}}}{m_{e}}\right),
\end{aligned}
$$

where $E_{e^{+}}, p_{e^{+}}$are the energy and momentum of an incident positron, respectively; $m_{e}$ is the mass of a core electron; $\mathrm{Z}$ is the atomic number of the core electron; $\sigma_{0}$ is the cross-section of dissipation of the electron energy. Further, the monochromatic optical depth of the investigated gas-and-dust medium should be expressed as follows:

$$
\tau_{\gamma}=n \sigma_{\mathrm{e}^{+}}^{+} \Delta \mathrm{x}
$$

where $\mathrm{n}$ is the gas density; $\Delta x$ is the thickness of the absorbing layer. In order to derive the $\gamma$-ray spectra of young supernova remnants and active galactic nuclei, as well as to perform their mapping, the obtained values $\tau_{\gamma}$ should be plugged in formulae (8) и (9). The dependence of the cross-section $\sigma_{e^{+}}^{+}$on the nucleus charge (expressed in relativistic units and equal to the atomic number of the chemical element Z) is illustrated in Table 1. The units of measurement for the cross-section are $\mathrm{cm}^{2}$. The crosssection for the light elements is comparable to the mean geometric cross-section of the K-shells of the target atoms. However, for the iron-peak elements the probability of positron annihilation with an L-electron is $10 \%$.

Next, we should take into account that when a positron slows down to the K-electron velocity, the single-photon annihilation with a K-electron occurs. In this case, the set of equations of conservation of energy and momentum can be written as follows: 
Table 1: Dynamic characteristics of the elements relevant to the employment of positron annihilation spectroscopy methods for the one-photon annihilation of positrons and $\mathrm{K}$-electrons in young supernova remnants

\begin{tabular}{|c|l|l|l|l|l|}
\hline El-nt & \multicolumn{1}{|c|}{ At. Wgt } & \multicolumn{1}{|c|}{$\mathrm{Eb}(\mathrm{eV})$} & \multicolumn{1}{|c|}{$\mathrm{Lg} \sigma_{e^{+}}^{+}$} & $E_{\gamma}(\mathrm{MeV})$ & $E_{n}(\mathrm{MeV})$ \\
\hline${ }_{1}^{1} \mathrm{H}$ & 1.0081 & 13.602 & -26.8551 & 1.026533 & 0.000564 \\
\hline${ }_{1}^{2} \mathrm{H}$ & 2.01410178 & 13.602 & -26.8551 & 1.026629 & 0.000467 \\
\hline${ }_{2}^{3} \mathrm{He}$ & 3.01602932 & 24.586 & -25.6339 & 1.026818 & 0.000267 \\
\hline${ }_{2}^{4} \mathrm{He}$ & 4.00260325 & 24.587 & -25.634 & 1.026873 & 0.000213 \\
\hline${ }_{3}^{6} \mathrm{Li}$ & 6.01512280 & 64.40 & -25.2194 & 1.026932 & 0.000114 \\
\hline${ }_{3}^{7} \mathrm{Li}$ & 7.01600456 & 64.40 & -25.2194 & 1.026912 & 0.000134 \\
\hline${ }_{4}^{7} \mathrm{Be}$ & 7.01692983 & 123,6 & -24.9133 & 1.026908 & $7.84 \mathrm{E}-05$ \\
\hline${ }_{4}^{9} \mathrm{Be}$ & 9.01218224 & 123,6 & -24.9133 & 1.026908 & $7.84 \mathrm{E}-05$ \\
\hline${ }_{6}^{12} \mathrm{C}$ & 12.0 & 296 & -24.4648 & 1.026767 & $4.74 \mathrm{E}-05$ \\
\hline${ }_{6}^{13} \mathrm{C}$ & 13.0033548 & 296 & -24.4648 & 1.026783 & $3.1 \mathrm{E}-05$ \\
\hline${ }_{7}^{14} \mathrm{~N}$ & 14.0030740 & 403 & -24.2843 & 1.021399 & 0.005308 \\
\hline${ }_{7}^{15} \mathrm{~N}$ & 15.0001089 & 403 & -24.2843 & 1.025435 & 0.001272 \\
\hline${ }_{8}^{16} \mathrm{O}$ & 15.9949146 & 538 & -24.1397 & 1.025731 & 0.000841 \\
\hline${ }_{8}^{17} \mathrm{O}$ & 16.9991317 & 538 & -24.1397 & 1.026539 & $3.34 \mathrm{E}-05$ \\
\hline
\end{tabular}

$$
\left\{\begin{array}{c}
E_{p}+2 m_{e} c^{2}=E_{\gamma}+E_{n}+E_{b} \\
P_{p}=P_{\gamma}+P_{n}
\end{array}\right.
$$

Alternatively, by squaring the second equation (the equation of conservation of momentum) and replacing the momentum moduli with the appropriate values of energy and mass from the expression $P^{2}=2 m E$, we obtain a set of equations which is more convenient for the calculations:

$$
\left\{\begin{array}{c}
E_{p}+2 m_{e} c^{2}=E_{\gamma}+E_{n}+E_{b} \\
2 m_{e} E_{p}=\left(\frac{E_{\gamma}}{c}\right)^{2}+2\left\lfloor\frac{E_{\gamma}}{c}\right\rfloor \sqrt{2 m_{n} E_{n}} \cos \alpha+2 m_{n} E_{n}
\end{array}\right.
$$

Here $E_{p}, E_{b}$ are the incident positron energy and binding energy of a K-electron of the target atom, respectively; $m_{e}$ is the electron (positron) mass; $E_{\gamma}$ is the sought value of the energy of the $\gamma$-quantum emitted upon annihilation; $E_{n}$ is the recoil energy of the nucleus. In the second equation of the set of equations (14), $\alpha$ is the emission angle between the atom and $\gamma$-quantum upon the annihilation of a positron and a $\mathrm{K}$-electron. In the investigated positron low-energy limit, the angles $\alpha$ range from 0 to $\pi / 2 \mathrm{rad}$. Then, the mean value of $\cos \alpha=1 / 2$.

The cross-sections for the annihilation of positrons and $\mathrm{K}$ - electrons are the greatest when their kinetic energies are equal to $E_{b}$. The numerical solution to the set of equations (11) is four pair of values for the recoil momentum of the nucleus $P_{n}$ and frequancies $v_{\gamma}$ of the emergent $\gamma$ quanta. The equation presented below was derived with adoption of the Hartree atomic unit system Hartree (1957). The electron or positron rest energy $E=m_{e} c^{2}=1$ was chosen as a unit of energy. The other physical quantities expressed in relativistic units are defined by the sequence of relations $\mathrm{h}=\mathrm{c}=\mathrm{m}_{\mathrm{e}}=1$. Finally, we will solve the following set of equations:

$$
\left\{\begin{array}{c}
E_{p}+2=E_{\gamma}+E_{n}+E_{b} \\
2 E_{p}=E_{\gamma}{ }^{2}+\left\lfloor E_{\gamma}\right\rfloor \sqrt{2 m_{n} E_{n}}+2 m_{n} E_{n}
\end{array} .\right.
$$

In case of positron deflection (scattering) at small angles $\approx 0 \mathrm{rad}$, the previous set of equations shall be re-written as follows:

$$
\left\{\begin{array}{c}
E_{p}+2=E_{\gamma}+E_{n}+E_{b} \\
2 E_{p}=E_{\gamma}{ }^{2}+2\left\lfloor E_{\gamma}\right\rfloor \sqrt{2 m_{n} E_{n}}+2 m_{n} E_{n}
\end{array} .\right.
$$

The solution for this set of equations will be expressed as follows:

$$
A E_{\gamma}^{4}+B E_{\gamma}^{3}+C E_{\gamma}^{2}+D E_{\gamma}+E=0
$$

Here $\quad A=1 ; B=2 m_{n} ; C=4\left[\left(m_{n}^{2}-m_{n}\left(E_{p}+2-\right.\right.\right.$ $\left.\left.E_{b}\right)-4 E_{p}\right] ; D=8 m_{n}\left(E_{p}-2 m_{n}\left(E_{p}+2-E_{b}\right)\right) ; E=$ $4\left[E_{p}-m_{n}\left(E_{p}+2-E_{b}\right)\right]^{2} ; m_{n}$ is the mass of the nucleus or its isotope; $E_{n}$ is the recoil energy of the given nucleus. Table 1 presents these values expressed in relativistic non-system units. In Table 1, the chemical elements produced in the supernova explosion and present in the SNR 1987A, as well as the respective values $E_{n}$ and $E_{\gamma}$ were adopted from the studies by Woosley (1991) and Popov et al. (2014). The obtained results allow not only comparing $P_{n}$ and $E_{\gamma}$ for the target atoms, but also detecting any correlation between the recoil momentum of nuclei and the associated Doppler broadening of their emission lines in the spectra of young supernova remnants and active galactic nuclei.

\section{Summary and discussion of the findings}

The following characteristic features of the radiative response described by Doikov et al. (2018) appear in the media exposed to the positron fluxes:

1. The presence of a characteristic non-thermal radiation field with its source function.

2. Non-thermal dual-mode electron energy distribution function associated with the injection of the Auger electrons into the medium.

3 . The presence of characteristic $\gamma$-lines in the spectrum of the nebular ejecta.

4. The presence of a correlation between the energies of $\gamma$-quanta, binding energies of nuclei and their recoil energies upon the single-photon annihilation of K-electrons in the respective atoms and incident positrons.

5. The calculations by formulae (16) have yielded $\gamma$ quanta energies which are not consistent with the results of experiments in the one-photon annihilation and physical properties of the interacting quantum systems predicted by the Feynman diagrams; hence, their adoption in solving the indicated problems is not reasonable.

6. The calculations by formulae (17) are important for the description of young supernova remnants and active galactic nuclei as they show the relationship between the recoil energy of the nucleus $E_{n}$ and the energy of the emitted $\gamma$ - quantum $E_{\gamma}$ given the energy of the incident positron of $0.01 \mathrm{MeV}$. The given values $E_{\gamma}$ are physically significant.

\section{Molecular spectra}

Active radio observations of young supernova remnants have been carried out for a long time, which allowed collecting extensive observational data. Potter et al. (2015) 
reported data on the strengthening of the fluxes of radio emission from the $\mathrm{H}_{2}$ molecule generated in the supernova ejecta. Generation of such fluxes requires the fulfilment of two conditions, such as sufficiently high amount of molecular hydrogen and the presence of an efficient source of energy. The first condition was met just after a three-year period of recombination of atomic hydrogen. With regard to the sources of excitation, the mapping of the images has shown that a source of hydrogen excitation is located in the supernova ejecta. As mentioned above, either positrons or the Auger electrons may serve as such a source. Non-optical photons are likely to destroy molecules. The Auger electrons or positrons should have energies of the order of $100 \mathrm{eV}$ to be able to excite the molecular hydrogen lines. The probability of the presence of positrons with such energies is low since as early as at such energies they efficiently annihilate with bound atomic electrons. In contrast, according to the functions of the electron energy distribution in the supernova ejecta $F(E, r)$ reported by Doikov et al. (2018), the likelihood of the presence of the Auger electrons with such energies is high. In these functions, $\mathrm{E}$ is the energy of electrons, and $\mathrm{r}$ is the current position of the examined unit volume of the ejecta relative to the explosion site. The increased emission flux from the $\mathrm{H}_{2}$ molecule confirms a substantial increase in the density (and proportion) of the Auger electrons with the indicated energies.

\section{Conclusions}

The presence of positron fluxes in the gas-and-dust aggregates of supernova remnants allows detecting their luminosity across various spectral bands.

1. The decay of proton-rich nuclei of the radioactive isotope ${ }_{22}^{44} \mathrm{Ti}$ via a series of transformations ${ }_{22}^{44} \mathrm{Ti} \stackrel{85 \mathrm{y}}{\longrightarrow}{ }_{21}^{44} \mathrm{Sc} \rightarrow{ }_{20}^{6 h} \mathrm{Ca}$ is a source of positrons in the supernova ejecta. The energy loss of positrons due to ionisation results in the creation of vacant $\mathrm{K}$ energy levels in atoms, followed by cascade transitions and ejecting of the Auger electrons. The hard UV and soft X-ray radiation field is formed; this radiation promotes further excitation of atoms and molecules.

2. Positron annihilation in the ejecta dust particles yields two $\gamma$-quanta each with the energy of $0.511 \mathrm{MeV}$.

3 . If the positron energy $E_{p}$ is close to the binding en$\operatorname{ergy} E_{b}$ of the atomic K-electron, the annihilation occurs with the emission of a single photon upon which the nucleus gains the recoil energy $E_{n}$. The data given in Table 1 enable to estimate the kinetic energy of atoms gained in the annihilation events.

4. The Doppler broadening is expected for the spectral lines of atoms involved in both radioactive decay and annihilation processes.

5. The cross-sections for annihilation with the emission of a single photon for various atoms of astrophysical relevance were obtained. From the preliminary determined cross-sections and De Broglie wavelengths for positrons, we have deduced which atomic electrons enter into annihilation with positrons.

6 . The author suggests considering the established correlation between $E_{n}$ and $E_{\gamma}$ to be a basis for the determi- nation of the chemical composition of the target supernova remnants and active galactic nuclei.

7. Using accurate positions of the $\gamma$-lines, it is feasible to specify the velocity range for positrons which contribute to these lines.

In summary, this study has shown the relationship between manifestations of the positron motion in the $\gamma$ region of the spectrum and the appropriate characteristics of the radiation field in cold gas-and-dust aggregates.

\section{References}

Grebenev S.A., Lutovinov, A.A., Tsygankov, S.S.: 2012, Nature, 490, 373.

Jerkstrand A., Branson C. and Korma C. T: 2011, A.\& Ap., 530, A45, pp. 1-23.

Kaastra J.S. \& Mewe R.: 1993, A \& Ap. Sp. Ser., 97, 443.

Hayakava S. Cosmic Ray Physics. Nuclear and Astrophysics Aspects. John Wiley and Sons. New York, 1969.

Anshu S., Helmana S., Priyanka A.: 2010, Applied Physics Research, 2, No 2, 176.

Gould R. J.: 1990, Ap.J., 362, 284.

Mikhailov A.I., Mikhailov I.A.: 1998, JETP, 113, N3, 786.

Doikov D.N., Andrievsky S.M., Yushchenko A.V.: 2018 , Journ. of Phys. Studies, 22, 2.

Doikov D.N., Andrievsky S.M.: 2017, Odessa Astron. Publ., 30, 63.

Doikov D.N., Andrievsky S.M., Yushchenko A.V.: 2018, Phys. Sciences and Technology, 5, No1, 60.

Potter T.M., Staneley-Smith L., Reville B. et al.: 2014, Ap. J., 794, 174.

Varosi F., Dwek E.: 1999, Ap. J., 523, 265.

Lucy, L. B., Danziger I. J., Gouiffes C. et al: 1991, in Supernovae, ed. Woosley (N.Y., Springer), 82.

Popov M.V., Filina A.A., Baranov A.A., Chardonet P. and V.M.Chechetkin: 2014, Ap. J., 783, 43.

Woosley S.E. \& Hoffman R.D.: 1991, Ap. J., 308, L31.

Hartree D.R. The calculation of atomic structures. (1957), John Wiley and Sons, New York. 251p.

Ferland et al.: 1998, PASP., 110, 749-761.

Meleh B.: 2014, Full doctor dissertation, Lviv University. 